\title{
Major signal increase in fluorescence microscopy through dark-state relaxation
}

\author{
Gerald Donnert, Christian Eggeling \& Stefan W Hell
}

\begin{abstract}
We report a substantial signal gain in fluorescence microscopy by ensuring that transient molecular dark states with lifetimes $>1 \mu \mathrm{s}$, such as the triplet state relax between two molecular absorption events. For GFP and Rhodamine dye Atto532, we observed a 5-25-fold increase in total fluorescence yield before molecular bleaching when strong continuous-wave or highrepetition-rate pulsed illumination was replaced with pulses featuring temporal pulse separation $>1 \mu \mathrm{s}$. The signal gain was observed both for one- and two-photon excitation. Obeying dark or triplet state relaxation in the illumination process signifies a major step toward imaging with low photobleaching and strong fluorescence fluxes.
\end{abstract}

As it draws on a myriad of markers and is noninvasive, fluorescence microscopy can be used to access the interior of cells with unsurpassed specificity ${ }^{1}$. Nevertheless, two key properties of standard fluorescence microscopy, namely the spatial resolution and the limited signal, call for major improvements ${ }^{2}$. Motivated by the first problem ${ }^{3}$, we addressed the latter and uncovered an illumination scheme that increases the fluorescence yield substantially.

To maximize fluorescence emission within a given time span, it is generally desirable to apply high excitation intensities. Besides, high intensities are mandatory in multiphoton microscopy ${ }^{4}$. However, intense excitation results in both enhanced triplet buildup and photobleaching, and thus in losses in the fluorescence signal. Therefore, excitation times of less than a microsecond readily elicit a substantial triplet population, which suggests the use of fast scanning in conventional confocal microscopes ${ }^{5-7}$. We now show that the total number of photons emitted by a dye that is subject to intense illumination substantially increases when one ensures that fluorophores caught in an absorbing dark state, such as the triplet state, are not further excited. Consequently, judicious pulsed excitation allowing for dark or triplet state relaxation (D-Rex or T-Rex), yields a lot more photons from the sample. This observation is relevant not only to microscopy but also to a range of bioanalytical techniques that, relying on fluorescence detection, are challenged by photobleaching.

Pulsed excitation is preferred or even required for imaging modalities such as fluorescence-lifetime imaging, stimulated emission depletion microscopy ${ }^{8}$ and multiphoton microscopy ${ }^{4}$.
In basically all of these modalities, pulse trains with a repetition rate $f=40-100 \mathrm{MHz}$ are used ${ }^{2}$. This rate is provided by most pulsed lasers, for example, by a mode-locked Ti:sapphire laser operating at $f=80 \mathrm{MHz}$. Moreover, the associated 'illumination pause' $\Delta t=1 / f$ of 10-25 ns between succeeding pulses is up to ten times larger than the typical lifetime of the fluorescent state $S_{1}$ of organic fluorophores and fluorescent proteins. Shorter $\Delta t$ increase the probability of illuminating molecules that are already excited, whereas longer $\Delta t$ would leave the dye idle. Hence, not surprisingly, increasing $\Delta t$ by decreasing $f$ appeared unattractive.

Exceptions to applying $f=40-100 \mathrm{MHz}$ have been rarely reported. For example, an amplified Ti:sapphire system emitting at $f=0.2 \mathrm{MHz}$ has been used in two-photon microscopy to benefit from the 400 times more intense pulses ${ }^{9}$, allowing deeper penetration into live brain ${ }^{10,11}$ or into tissue ${ }^{12}$. In another experiment, wide-field one-photon excitation with a pulsed laser diode at $f=6 \mathrm{kHz}$ has been used to saturate the fluorescent $S_{1}$ state and for technical convenience ${ }^{13}$. But having relied on a single frequency, these studies could not reveal the relationship between $\Delta t$ and the signal magnitude. We found that intermissions $\Delta t$ of $\sim 0.5-2 \mu \mathrm{s}$ boost the number of photons emitted from a molecule before bleaching. This should facilitate a number of applications that have so far been curbed by a lack of signal. This finding holds both for intense one- and inherently intense two-photon excitation.

\section{RESULTS}

\section{Experimental approach}

We studied the role of dark or triplet state relaxation in pulsedmode illumination by gradually reducing the repetition rate $f$ from $40 \mathrm{MHz}$ down to $0.5 \mathrm{MHz}$, which is equivalent to expanding the inter-pulse distance $\Delta t$ from 25 ns to $2 \mu \mathrm{s}$. We selected two fundamentally different fluorophores, Atto532 and GFP. The first is a Rhodamine dye known for its photostability, low intersystem crossing to the triplet state $(<1 \%)$ and high fluorescence yield $^{3,5,14}$. GFP is the archetype of the genetically encodable fluorescence markers ${ }^{15}$.

We prepared the fluorophores as thin layers on a glass cover slip and covered them with the mounting medium Mowiol. This measure avoided molecular diffusion as a source of signal replenishment during photobleaching. To quantify photobleaching,

Max Planck Institute for Biophysical Chemistry, Department of NanoBiophotonics, Am Fassberg 11, 37077 Göttingen, Germany. Correspondence should be addressed to S.W.H. (shell@gwdg.de).

RECEIVED 4 AUGUST; ACCEPTED 31 OCTOBER; PUBLISHED ONLINE 10 DECEMBER 2006; DOI:10.1038/NMETH986 

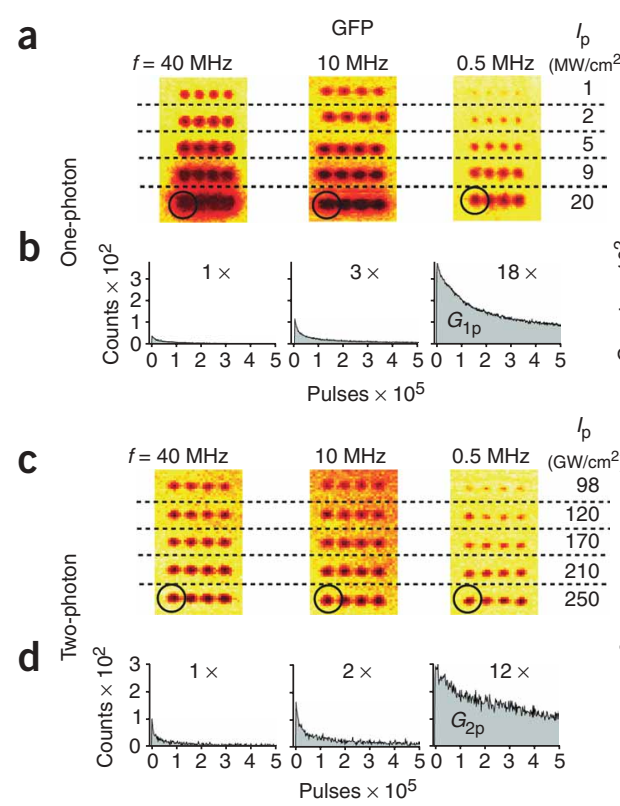
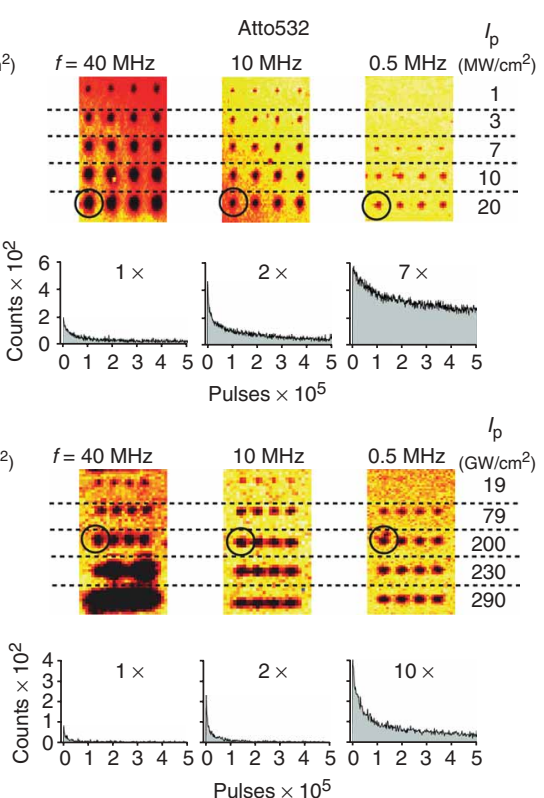

Figure 1 | Photobleaching of GFP and Att0532 decreases with inter-pulse break $\Delta t=1 / f$ for oneand two-photon excitation. (a-d) Fluorescence images of the fluorophore layers $(7 \times 10.5 \mu \mathrm{m})$ after illumination with the indicated repetition rates $f$ (vertically aligned) and pulse-peak intensities, $I_{\mathrm{p}}$ (horizontally aligned, dashed lines; a,c). For each pair $f$ and $I_{p}$, four runs were recorded, arrayed in the same row. The number of excitation pulses was $2.8 \times 10^{6}$ for all exposures (1.4 $\times 10^{6}$ for one-photon excitation of Atto532). Image recording after spot bleaching was done with very low intensities at $40 \mathrm{MHz}$, the scale being the same for GFP and Att0532. Dark areas signify the spots at which the signal was recorded, with the darkness being a measure of the bleaching that occurred. The decrease in fluorescence $(\mathbf{b}, \mathbf{d})$ observed at the spots during irradiation, circled in $\mathbf{a}$ and $\mathbf{c}$, its integral giving the total signal $G_{1 p}$ and $G_{2 p}$ for one- and two-photon excitation, respectively. Note the increase of the initial peak and of $G_{1 \mathrm{p}}$ and $G_{2 \mathrm{p}}$ with increasing $\Delta t$. The magnification factors indicate the relative increase of $G_{1}$ and $G_{2 p}$ with decreasing $f$. we irradiated the layers with a stationary beam focused to a $\sim 200$-nm-diameter spot for a certain number of pulses and imaged the photobleached spots after completion of illumination (Fig. 1). We changed both the repetition rate $f$ and the pulse-peak intensity $I_{\mathrm{p}}$ of irradiation. We applied five values of $I_{\mathrm{p}}$ both for oneand two-photon excitation. For each pair $f$ and $I_{\mathrm{p}}$, we recorded four runs. Recording the fluorescence as a function of time indicated the photobleaching rate, whereas the integration of the fluorescence over time disclosed the total signal $G_{1 p}$ and $G_{2 p}$ gained by one- and two-photon excitation, respectively. Notably, all runs to be compared were performed with the same total number of pulses, irrespective of the repetition rate, because we compensated the reduction of $f$ by concomitantly extending the exposure time.

\section{Fluorescence gain for one-photon excitation}

The total illumination time for one-photon excitation (470 nm, pulse width $\tau_{\mathrm{P}}=100 \mathrm{ps}$ ) of GFP at $f=40 \mathrm{MHz}$, $10 \mathrm{MHz}$ and $0.5 \mathrm{MHz}$ was $70 \mathrm{~ms}, 280 \mathrm{~ms}$ and $5.6 \mathrm{~s}$, respectively, implying that the spots were exposed to $2.8 \times 10^{6}$ pulses. For

Figure 2 | Total fluorescence signal generated by one-photon excitation $\left(G_{1 \mathrm{p}}\right)$ for GFP and Atto532 for a given number of excitation pulses $\left(1.4 \times 10^{6}\right)$. (a) $G_{1 \mathrm{p}}$ as a function of $\Delta t=1 / f$. The magnification factors indicate the relative increase in total fluorescence. The s.d. (error bars, $G_{1 p} \pm 2-5 \%$ of the total signal) were determined from five replicate measurements. The solid lines represent an exponential fit to the data, mimicking the extent of the decay of a nonfluorescent dark (triplet) state of 1- $\mu$ s lifetime between two subsequent pulses. Note that an optimal gain in $G_{1 \mathrm{p}}$ is predicted for $f \approx 100 \mathrm{kHz}$, close to the smallest applied rate of $500 \mathrm{kHz}$. In the case of a 5 - $\mu$ s lifetime (dashed line), the optimal frequency shifts to lower repetition rates. (b) $G_{1 p}$ as a function of the pulse peak intensity $I_{\mathrm{p}}$ with $\Delta t$ as parameter. The number of excitation photons and illumination time of continuous-wave (CW) illumination was the same as that of its $40-\mathrm{MHz}$ pulsed counterpart. For strong excitation intensities, pulsed excitation with $\Delta t>1 \mu$ s lead to higher total fluorescence yield per fluorescent molecule. The vertical dashed lines outline the intensity $I_{\mathrm{p}}$ used in a.
Atto532, the number of excitation pulses was $1.4 \times 10^{6}$ and the illumination time thus halved.

Photobleaching increased with increasing $I_{\mathrm{p}}$ (Fig. 1a). Although this finding arguably may have been expected ${ }^{14}$, less expected was the fact that photobleaching is substantially weaker at the lower $f$.

We plotted the fluorescence recorded during irradiation as a function of the number of excitation pulses applied (Fig. 1b). In fact, these curves quantify the initial fluorescence strength along with its decrease for the first $5 \times 10^{5}$ pulses. Each data point represents the signal accumulated from 2,000 pulses ( 1,000 pulses for one-photon excitation of Atto532). Being recorded at $f=40,10$ and $0.5 \mathrm{MHz}$, the curves reveal that increasing $\Delta t$ increases the signal dramatically. We observed the increase both for the initial signal generated by the first 1,000-2,000 pulses applied to the spot (note the initial peak in Fig. 1b) and for the integrated onephoton-generated signal $G_{1 \mathrm{p}}$. For example, the curves in Figure $\mathbf{1 b}$ indicate that the reduction of the repetition rate from $f=40 \mathrm{MHz}$ to $0.5 \mathrm{MHz}$ for one-photon excitation with $I_{\mathrm{p}} \approx 20 \mathrm{MW} / \mathrm{cm}^{2}$ increases the $G_{1 p}$ from GFP by 18 -fold and that from Atto532 by
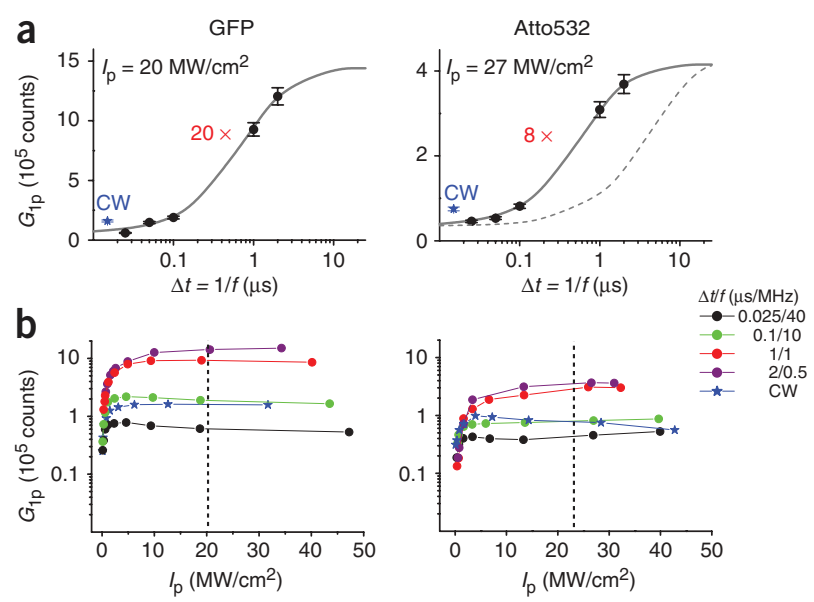


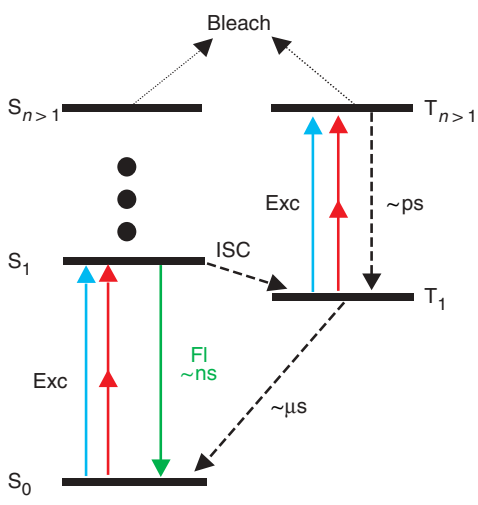

Figure 3 | Energy diagram of a typical organic fluorophore, indicating the major molecular pathways for excitation (Exc), fluorescence (Fl), intersystem crossing (ISC), relaxation (dashed lines) and photobleaching (bleach). Singlet states: $S_{0}, S_{1}, S_{n>1}$; triplet states: $T_{1}, T_{n}>1$. Owing to the chemical fragility of the triplet system, the excitation of $T_{1}$ is an effective bleaching pathway. Although the lifetime of the fluorescent state $S_{1}$ is $\sim 1-4 \mathrm{~ns}$, that of the vulnerable $\mathrm{T}_{1}$ is longer by three orders of magnitude: $\sim 1 \mu \mathrm{s}$.

sevenfold. The actual improvement is larger because the curves in Figure $1 \mathbf{b}$ just show the signal for the first $5 \times 10^{5}$ pulses. Plotting the signal $G_{1 p}$ obtained from $1.4 \times 10^{6}$ pulses as a function of $\Delta t$ (Fig. 2a) reveals that the signal improvement actually is 20 -fold for GFP and eightfold for Atto532.

We plotted $G_{1 p}$ as a function of $I_{\mathrm{p}}$ ranging up to $50 \mathrm{MW} / \mathrm{cm}^{2}$ (Fig. 2b); here we used $\Delta t=1 / f$ as a parameter. For strong excitation intensities $I_{\mathrm{p}}$, the maximum $G_{\mathrm{lp}}$ was attained at the highest $\Delta t$. These results underscore that $\Delta t$ has a decisive role in keeping the dye fluorescent. Moreover, the most substantial increase in $G_{1 \mathrm{p}}$ occurs between $\Delta t=0.1$ and $1 \mu$ s. This observation suggests that the precursor of photobleaching of Atto532 and GFP is a dark state with a lifetime of $\sim 1 \mu$ s (Fig. 2a). Increasing $\Delta t$ allows the spontaneous relaxation of this state between two excitation pulses, thereby avoiding its pile-up and its excitation to a further reactive state ${ }^{16,17}$. The primary candidate for this dark state is the molecular triplet state $T_{1}$ because in most fluorophores the $T_{1}$ relaxes within $\sim 1 \mu$ s under ambient conditions ${ }^{18}$. We illustrate these states and the associated pathways of excitation and photobleaching in Figure 3.

It is now interesting to compare the $G_{1 p}$ values found for the pulsed illumination with those for continuous-wave exposure (Fig. 2). As there is no dedicated intermission in the continuouswave case, the population of the dark state and its excitation just depend on the continuously applied intensity $I_{\mathrm{CW}}$. The continuous-

Figure 4 | Total fluorescence signal generated by two-photon excitation $\left(G_{2 p}\right)$ for GFP and Atto532 for a given number of excitation pulses $\left(1.4 \times 10^{6}\right)$. (a) $G_{2 p}$ as a function of $\Delta t=1 / f$. The magnification factors indicate the relative increase in total fluorescence. The s.d. (error bars, $G_{2 p} \pm 4-6 \%$ of the total signal) were determined from five repetitive measurements. The gray lines reveal the extent of the decay of a nonfluorescent dark (triplet) state of $1 \mu \mathrm{s}$ (solid line) and $5 \mu \mathrm{s}$ (dashed line) lifetime in between subsequent pulses. (b) $G_{2 p}$ as a function of the pulse peak intensity $I_{\mathrm{p}}$ with $\Delta t$ as parameter. The vertical line outlines the intensity $I_{p}$ used in a. (c) Ratio of one- and two-photon-generated fluorescence $\left(G_{2 p} / G_{1 p}\right)$ as a function of $\Delta t$, showing that the total fluorescence yield is lower for two-photon excitation than for its one-photon excitation counterpart, but the ratio decreases by twofold for $\Delta t>1 \mu \mathrm{s}$. wave data were obtained by illuminating for the same illumination time and with the same time-averaged intensity $I_{\mathrm{CW}}=I_{\mathrm{p}}\left(\tau_{\mathrm{p}} f\right)$ as in the $40 \mathrm{MHz}$ case. The latter condition is like spreading out the photons of a pulse across the 25 -ns break. At the $488 \mathrm{~nm}$ wavelength used for continuous-wave illumination, the absorption cross-section is higher than at the $470 \mathrm{~nm}$ wavelength used for pulsed illumination. Nevertheless, for strong intensities, the fluorescence signal gained by the continuous-wave strategy was by up to fivefold (GFP) and by up to eightfold (Atto532) smaller than in the $0.5 \mathrm{MHz}$ pulsed case. Note, that the maximum applied intensities of up to $I_{\mathrm{CW}}$ of $\sim 200 \mathrm{~kW} / \mathrm{cm}^{2}$ were about an order of magnitude above those usually applied in fluorescence microscopy ${ }^{2,16}$, but they were in the range of what is routinely used in single-molecule experiments in solution ${ }^{17}$. Hence, an intense continuous-wave illumination cannot provide the same total signal that is provided by pulsed D-Rex illumination.

\section{Fluorescence gain for two-photon excitation}

We measured the total fluorescence signal generated by two-photon excitation ( $800 \mathrm{~nm}$, pulse width $\tau_{\mathrm{p}}=200 \mathrm{fs} ;$ Fig. 4). Like in the onephoton mode, the total number of pulses was $1.4 \times 10^{6}$ in all cases. For pulse peak intensities $I_{\mathrm{p}} \approx 300 \mathrm{GW} / \mathrm{cm}^{2}$, about 1.5-5-fold larger than usually applied in fluorescence microscopy ${ }^{2,4,16}$, enlarging $\Delta t$ from $25 \mathrm{~ns}$ to $2 \mu$ s increased the total two-photon fluorescence yield $G_{2 p}$ by $\sim 25$-fold for GFP and $\sim 20$-fold for Atto532.

We plotted $G_{2 p}$ as a function of $I_{\mathrm{p}}$, with $\Delta t=1 / f$ as parameter (Fig. $\mathbf{4 b}$ ). Again, $G_{2 p}$ became saturated with increasing $I_{\mathrm{p}}$; the saturation level strongly depended on $\Delta t$. Larger $\Delta t$ allowed more emissions and once more, both GFP and Atto532 exhibited the decisive increase in $G_{2 p}$ when stepping up from $\Delta t=0.1$ to $1 \mu$ s. This finding showed again that a $\sim 1-\mu$ s dark
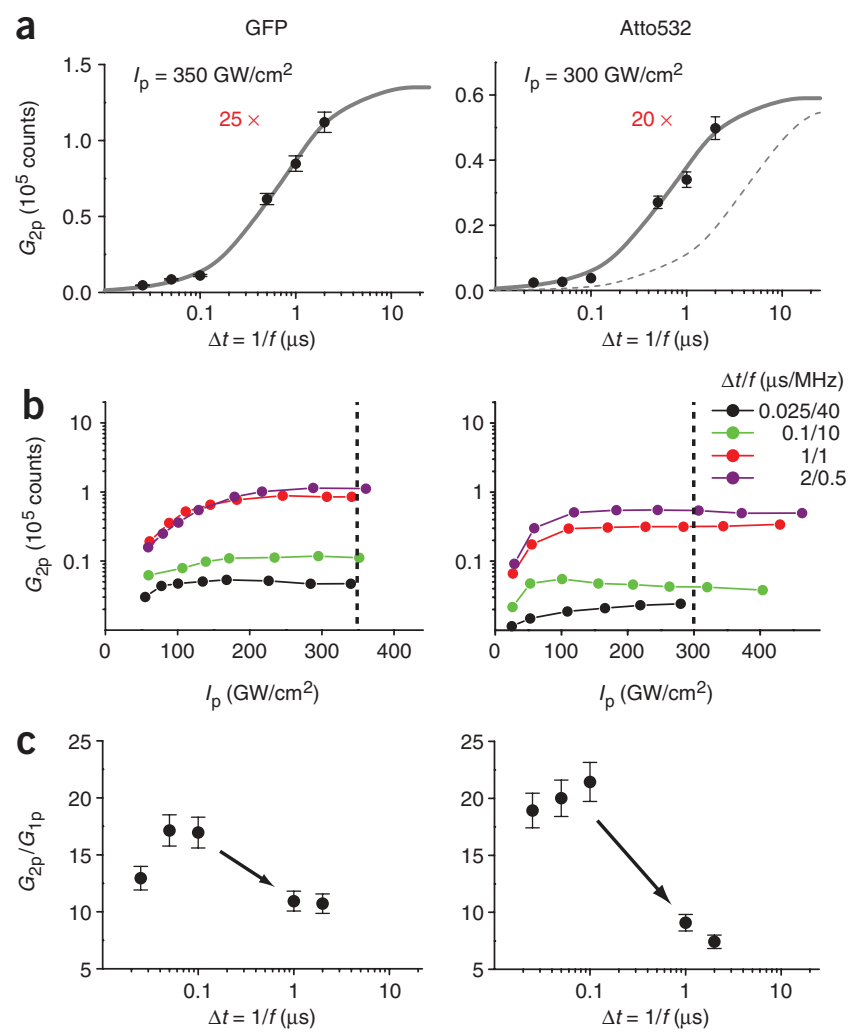


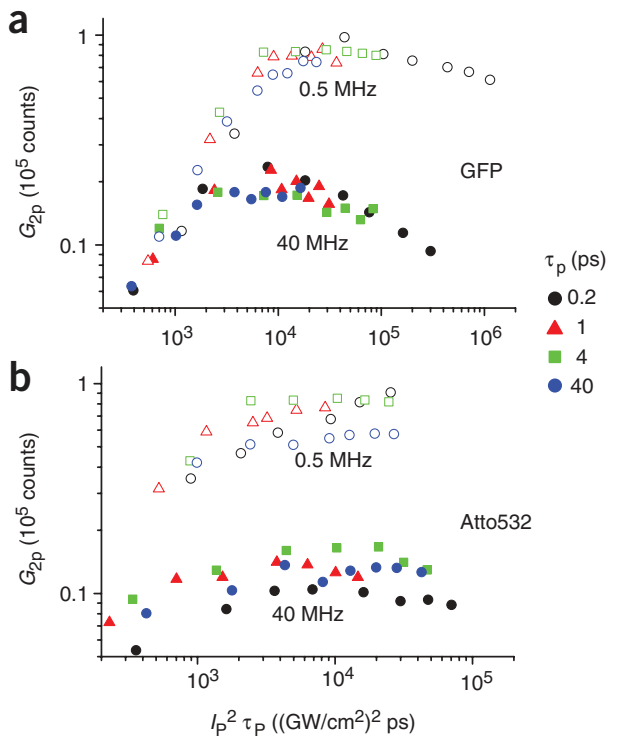

Figure 5 | The pulse duration does not affect the total fluorescence signal $G_{2 p}$ of two-photon excitation. $(\mathbf{a}, \mathbf{b})$ Dependence of photobleaching on the pulse width $\left(\tau_{\mathrm{p}}\right)$ for two-photon excitation of GFP (a) and Atto532 (b). The curves show $G_{2 p}$ for $0.5 \mathrm{MHz}$ (open) and $40 \mathrm{MHz}$ (filled) with increasing value of $I_{\mathrm{p}}{ }^{2}$ $\tau_{p}$ giving the ability of a pulse to excite in the two-photon mode. The latter is maintained when varying the pulse widths from $\tau_{\mathrm{p}}=200 \mathrm{fs}$ to $40 \mathrm{ps}$. Pulses that are longer by $m$ feature a pulse energy that is larger by $\sqrt{ } m$ and an $I_{\mathrm{p}}$ that is lower by the same factor.

state had a key role in the fluorescence bleaching (and saturation) mechanism (Fig. 4a).

This similarity has led us to compare the total yield for both oneand two-photon excitation modes. This comparison is interesting because two-photon excitation reportedly involves stronger photobleaching ${ }^{16,19,20}$. Therefore, we selected $I_{\mathrm{p}}=25 \mathrm{MW} / \mathrm{cm}^{2}$ and $300 \mathrm{GW} / \mathrm{cm}^{2}$ as pulse peak intensities, yielding the maximum possible fluorescence signals $G_{1 p}$ and $G_{2 p}$, respectively, and plotted the ratio $G_{2 \mathrm{p}} / G_{1 \mathrm{p}}$ as a function of $\Delta t$ (Fig. 4c). We found that for $\Delta t \leq 0.1 \mu \mathrm{s}$, the $G_{2 \mathrm{p}}$ was indeed up to 20 times lower than $G_{1 \mathrm{p}}$, but this adverse factor was below tenfold at $\Delta t \geq 0.1 \mu \mathrm{s}$, thus narrowing the gap. This finding indicates that in a multiphoton excitation modality, the intermissions $\Delta t$ are even more effective.

In addition, this observation provides further evidence for dark state absorption because in the multiphoton mode, the pulse intensity is larger than that for its one-photon counterpart by several orders of magnitude. Once a molecule has passed to an absorbing dark state, such as the $\mathrm{T}_{1}$, the molecule is confronted with a photon flux that leads to a single (and possibly also multiphoton) excitation to the $T_{n>1}$ (see Fig. 3). These processes are catalyzed by the fact that the cross-section for a one-photon absorption of the $\mathrm{T}_{1}$ state is $\sigma_{\mathrm{T}} \approx 10^{-17} \mathrm{~cm}$ (refs. 2,21). Given $I_{\mathrm{p}}>50 \mathrm{GW} / \mathrm{cm}^{2}$ and a photon energy of $2.5 \times 10^{-19} \mathrm{~J}$, the excitation rate of a $\mathrm{T}_{1}$ molecule is $\sim 1$ event $\operatorname{per}\left(\tau_{\mathrm{p}} \approx 200 \mathrm{fs}\right)$ pulse, that is, the $\mathrm{T}_{1} \rightarrow \mathrm{T}_{n}>1$ process is nearly saturated. Therefore, once the molecule has crossed to the $T_{1}$, it is prone to be excited to a more fragile state. Furthermore, the $T_{n}>1$ molecule can absorb another photon from the same pulse, thus setting off a cascade of several consecutive one-photon excitations to increasingly fragile states. This observation explains why the D-Rex illumination modality is so effective.

In fact, we found other evidence for this characteristic when scrutinizing the role of the pulse duration $\tau_{\mathrm{p}}$ on $G_{2 \mathrm{p}}$. Accommodating $\tau_{\mathrm{p}}=0.2,1,4$ and 40 ps both for $f=40$ and $0.5 \mathrm{MHz}$ pulse trains, in Figure 5 we plotted $G_{2 p}$ as a function of $I_{\mathrm{p}}{ }^{2} \tau_{\mathrm{p}}$, which is proportional to the probability of two-photon excitation within a single pulse. Increasing $\tau_{\mathrm{p}}$ by a factor $m$ while keeping $I_{\mathrm{p}}{ }^{2}$ $\tau_{\mathrm{p}}$ constant, entails an $I_{\mathrm{p}}$ that is lower by $\sqrt{ } m$; the pulse energy $\left(\sim I_{\mathrm{p}} \tau_{\mathrm{p}}\right)$ increases by the same factor. Spanning over two orders of magnitudes, the variation in $\tau_{\mathrm{p}}$ exceeds by far the $100 \mathrm{fs}-2 \mathrm{ps}$ span encountered in practical two-photon microscopy ${ }^{4,22}$. The data shown in Figure $\mathbf{5}$ confirm that stepping down $f$ leads to a substantial increase in $G_{2 \mathrm{p}}$, but changing $\tau_{\mathrm{p}}$ by $m=200$ and hence the pulse energy by 14 -fold does not have a considerable effect on $G_{2 p}$. This obviously indicates that the saturation of the $\mathrm{S}_{0} \rightarrow \mathrm{S}_{1}$ or of the $\mathrm{T}_{1} \rightarrow \mathrm{T}_{n}>1$ excitation eliminates any dependence on pulse peak intensity or energy. Especially the saturation of $\mathrm{T}_{1} \rightarrow$ $\mathrm{T}_{n}>1$ is consistent with our findings: following a two-photon excitation to the $S_{1}$, the molecule crosses to the dark state (for example, the $\mathrm{T}_{1}$ ), which is then subject to a further nearly saturated excitation (for example, to $\mathrm{T}_{n}>1$ ). The saturation is either provoked by large $I_{\mathrm{p}}$ for short $\left(\tau_{\mathrm{p}}=200 \mathrm{fs}\right)$ or by repetitive excitation to $\mathrm{T}_{n>1}$ within the same long pulse $\left(\tau_{\mathrm{p}}=40 \mathrm{ps}\right)$ with the lifetime of $\mathrm{T}_{n>1}$ being $\sim 200 \mathrm{fs}$.

\section{Increased image brightness}

D-Rex illumination conditions yield brighter two-photon images of Escherichia coli cells expressing the Venus yellow fluorescent protein $^{23}$ (Fig. 6). In each recording, we applied $4 \times 10^{4}$ pulses of $I_{\mathrm{p}}=350 \mathrm{GW} / \mathrm{cm}^{2}$ per $62.5-\mathrm{nm}$ quadratic pixel but used different $\Delta t$. The brightness histogram revealed that the 40 -fold increase in $\Delta t$ provided an approximately threefold brighter image (Fig. 6c). In light of the fact that Venus is a member of the GFP family, the data in Figure 6 underscore the relevance of D-Rex for other fluorophores.

\footnotetext{
Figure 6 | Two-photon fluorescence images of Escherichia coli cells expressing the fluorescent protein Venus are brighter when recorded in the D-Rex mode. (a,b) Pulse intermissions $\Delta t=25$ ns (a) and $1 \mu \mathrm{s}(\mathbf{b})$. The same number of excitation pulses was ensured for both images by adjusting the exposure time accordingly (1 ms, a and $40 \mathrm{~ms}, \mathbf{b})$. Scale bar, $1 \mu \mathrm{m}$. (c) Histogram of the counts per pixel of the images in a (black) and $\mathbf{b}$ (red).
}
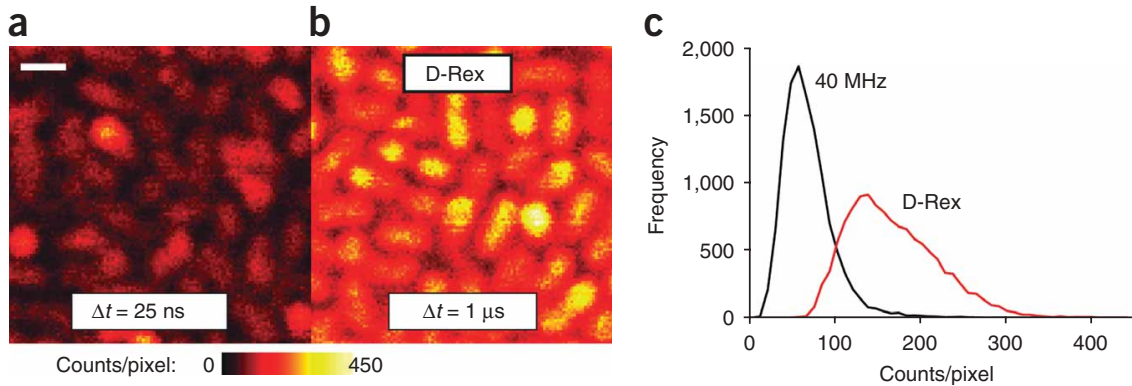


\section{DISCUSSION}

The total fluorescence generated by intense one- and two-photon excitation from a molecule can be increased by applying light pulses of a rate $f<1 \mathrm{MHz}$. Photobleaching is reduced, because intermissions $\Delta t=1 / f>1 \mu$ s between succeeding pulses permit the decay of an absorbing dark state of similar lifetime. We have argued that the primary candidate for this state is the triplet state $T_{1}$, because the $1-\mu$ s relaxation time coincides with the $T_{1}$ lifetime of most fluorophores under ambient conditions. Another reason is that the $T_{1}$ is a feature of organic fluorophores and fluorescent proteins alike. Although it has been known that the population of the $\mathrm{T}_{1}$ reduces the instant fluorescence flux ${ }^{2,6,7}$, our results showed that the population of such a state is critical to the total fluorescence yield and the photostability. Also, we observed the two-photon fluorescence yield $G_{2 p}$ to slightly vary with the sample preparation. For example, in the data presented in Figure 5, the gain for GFP is lower as compared to that in Figure 4, which was recorded on a sample that was ten days old. As the embedding medium Mowiol solidifies with time, the concentration and mobility of oxygen decrease, increasing the $\mathrm{T}_{1}$ lifetime. A shortened $\mathrm{T}_{1}$ lifetime lowers the $\mathrm{T}_{1}$ pile-up and abates the effect of $\mathrm{D}$-Rex microscopy. In contrast, an increased dark state lifetime will enforce $f<<1$ $\mathrm{MHz}$ (Figs. 2a and 4a). Hence, although the boost factor depends on the chemical environment, most-if not all-of the fluorophores are expected to exhibit gain under D-Rex conditions. In fact, ad hoc screening of the organic dyes Rhodamine6G, Coumarin120, DAPI and the enhanced yellow fluorescent protein have also exhibited a 5-15-fold fluorescence increase.

As dark-state photobleaching is reduced by D-Rex illumination, $\mathrm{S}_{1} \rightarrow \mathrm{S}_{n}>1^{-}$-induced photobleaching becomes more prominent. Besides, nonexcited $\mathrm{T}_{1}$ molecules may also bleach. D-Rex illumination is less important for one-photon excitation at low (continuous-wave) intensities, as can also be inferred from the $G_{1 p}$ data (Fig. 2b). Continuous-wave excitation at low intensities inherently fulfills D-Rex conditions, but implies a poor fluorescence rate. In contrast, in a typical two-photon microscope operating at $80 \mathrm{MHz}$ and scanning with a pixel dwell time of $5 \mu \mathrm{s}$, a molecule is hit by 400 pulses in a row at $\Delta t=12.5 \mathrm{~ns}$ with each pulse containing $\sim 10^{8}$ photons. Once it has passed to the $\mathrm{T}_{1}$, it is confronted with these pulses, explaining why even fluorophores with a low $(<1 \%)$ rate for $S_{1} \rightarrow T_{1}$ crossing, such as Atto532, exhibit a substantial gain by the D-Rex modality. Yet high intensities are also necessary in one-photon excitation to maximize signal and to cut down acquisition times. Our data indicate that D-Rex schemes allow the application of high intensities while still providing a high fluorescence yield.

Implementing D-Rex by decreasing $f$ is not optimal, because of the concurrent increase in recording time. For single-beam scanning, D-Rex may be limited to static samples, but widefield D-Rex illumination using $f<1 \mathrm{MHz}$ certainly can accommodate live-cell imaging and single-particle tracking, where both strong excitation and long-lasting signals are needed. Another implementation of the D-Rex modality is to scan the beam so fast that succeeding pulses illuminate different molecules, although image alignment during fast mirror scanning could be difficult. An elegant way of fast scanning is the application of spinning disks in which case synchronization does not pose problems. Another option is to combine low-repetition-rate scanning with parallelized multispot or line-shaped illumination. Our results corroborate anecdotal reports that fast-scanning microscopes involve less bleaching and that $f>100 \mathrm{MHz}$ lasers do not always provide the anticipated signal in multiphoton microscopy. We also note that unlike the addition of antifade chemical agents, the D-Rex strategy is allphysical and hence compatible with live cells.

In conclusion, the D-Rex illumination strategy increases the one- and two-photon excitation generated fluorescence yield. Moreover, it narrows the gap in brightness between the two excitation modes. Altogether, we expect that the D-Rex illumination scheme will improve many biotechnology applications that rely on fluorescence.

\section{METHODS}

Materials. The organic dye Atto532 NHS ester (emission 540$580 \mathrm{~nm}$ ), a Rhodamine derivative, was a kind gift from K.-H. Drexhage (University of Siegen). We realized a monolayer of Atto532 by coupling the dye to a silanized coverglass via the NHS linker. We analyzed purified GFP in an 500-nm-thick layer adsorbed on a cover glass. The layers were mounted by Mowiol (4.8 g Mowiol 4-88 (Fluka), $12 \mathrm{~g}$ glycerol, $12 \mathrm{ml}$ double-distilled water, $24 \mathrm{ml} \quad 0.2 \mathrm{M}$ Tris buffer ( $\mathrm{pH} 8$ ), $52.8 \mathrm{mg}$ Dabco (1,4-diazabicyclo[2,2,2] octan; Fluka)). The Venus fluorescent protein was expressed in Escherichia coli B121CodonPlus sustained on a poly(L-lysine)-coated cover glass.

Setup. We performed the experiments with a stage-scanning fluorescence microscope featuring an oil immersion objective (60× magnification, 1.4 numerical aperture, that is, $\alpha=68^{\circ}$; Leica) producing nearly diffraction-limited spots. For onephoton excitation we used a linearly polarized pulsed laser diode (470 nm, $\tau_{\mathrm{p}}=100 \mathrm{ps,} \mathrm{LDH-P-C-470;} \mathrm{PicoQuant} \mathrm{GmbH).} \mathrm{We}$ varied $f$ by means of a frequency generator. We used a linearly polarized argon laser (Omnichrome Series 43; Melles Griot) for continuous-wave excitation at $488 \mathrm{~nm}$. For two-photon excitation at $800 \mathrm{~nm}$, we used a femtosecond mode-locked, linearly polarized titanium:saphire laser of $80 \mathrm{MHz}$ repetition rate (MaiTai, Spectra-Physics Lasers $\mathrm{GmbH}$ ). We used a single-pulse selector (Pulse Select; APE $\mathrm{GmbH}$ ) equipped with a $\mathrm{TeO}_{2}$ Bragg cell to adjust $f$ from $40 \mathrm{MHz}$ down to $0.3 \mathrm{MHz}$. Where required, we stretched the initial pulse width $\tau_{\mathrm{p}}=200 \mathrm{fs}$ to $\sim 1$ ps by a 20 -cm-long SF6 glass rod. We realized longer durations by passing the pulses through 2-20-m-long glass fibers (PMF-RC-820-BL single-mode polarization-maintaining, cutoff at $820 \mathrm{~nm}$; StockerYale Inc.). We measured $\tau_{\mathrm{p}}$ with an autocorrelator (Pulse Check, APE GmbH) or a microchannel plate photomultiplier tube (R3809U, Hamamatsu). The fluorescence emission was collected by the same objective lens and projected onto an avalanche photodetector (SPCM-AQR-13-FC, Perkin Elmer Optoelectronics) with an aperture size corresponding to $0.8 \times$ the magnified Airy disk of the fluorescence spot. The detection events were further processed by a PC card (SPC 730, Becker \& Hickl GmbH), allowing observation of the fluorescence-count rate within varying observation time windows. We probed the intensity point-spread function (PSF) of the excitation laser by a gold bead of $80-\mathrm{nm}$ diameter on a nonconfocal detector (MP 963 Photon Counting Module, Perkin Elmer). The full width at half maximum (FWHM) of the PSF in the focal plane and the power $P$ measured at the sample entered the calculation of the applied pulse peak intensities $I_{\mathrm{p}}=P /\left(\pi(0.5 \mathrm{FWHM})^{2}\left(\tau_{\mathrm{p}} f\right)\right)$. 


\section{ACKNOWLEDGMENTS}

We thank A. Miyawaki (Laboratory for Cell Function and Dynamics, Riken) for supplying the fluorescent protein Venus. We also thank S. Jakobs, S. Verrier and D. Ouw for preparation of the samples, A. Giske, R. Kellner and K. Willig for help with the experimental setup, and V. Westphal and A. Egner for fruitful discussions. Finally, we thank A. Schönle for support with the IMSPECTOR software.

\section{COMPETING INTERESTS STATEMENT}

The authors declare that they have no competing financial interests.

\section{Published online at http://www.nature.com/naturemethods/}

Reprints and permissions information is available online at

http://npg.nature.com/reprintsandpermissions/

1. Tsien, R.Y. Imagining imaging's future. Nat. Rev. Mol. Cell Biol. 4 (Suppl.), SS16SS21 (2003).

2. Pawley, J.B. (ed.) Handbook of biological confocal microscopy $3^{\text {rd }}$ edn. (Springer, New York, 2006).

3. Donnert, G. et al. Macromolecular-scale resolution in biological fluorescence microscopy. Proc. Natl. Acad. Sci. USA 103, 11440-11445 (2006).

4. Denk, W., Strickler, J.H. \& Webb, W.W. Two-photon laser scanning fluorescence microscopy. Science 248, 73-76 (1990).

5. Tsien, R.Y., Ernst, L. \& Waggoner, A. Fluorophores for confocal microscopy: photophysics and photochemistry. in Handbook of biological confocal microscopy, $3^{\text {rd }}$ edn. (ed., Pawley, J.B.) 338-352 (Springer, New York, 2006).

6. Webb, W.W., Wells, K.S., Sandison, D.R. \& Strickler, J. Criteria for quantitative dynamical confocal fluorescence imaging. in Optical Microscopy for Biology (eds Herman, B. \& Jacobson, K.) 73-108 (Wiley, New York, 1990).

7. Conchello, J.-A. \& Lichtman, J.W. Optical sectioning microscopy. Nat. Methods 2 , 920-931 (2005).

8. Hell, S.W. \& Wichmann, J. Breaking the diffraction resolution limit by stimulated emission: stimulated emission depletion microscopy. Opt. Lett. 19, 780-782 (1994).

9. Brakenhoff, G.J. et al. Real-time two-photon confocal microscopy using a femtosecond, amplified Ti:sapphire system. J. Microsc. 181, 253-259 (1996).
10. Beaurepaire, E., Oheim, M. \& Mertz, J. Ultra-deep two-photon fluorescence excitation in turbid media. Opt. Commun. 188, 25-29 (2001).

11. Theer, P., Mazahir, H.T. \& Denk, W. Two-photon imaging to a depth of $1000 \mu \mathrm{m}$ in living brains by use of a Ti: $\mathrm{Al}_{2} \mathrm{O}_{3}$ regenerative amplifier. Opt. Lett. 28, 1022-1024 (2003).

12. Masters, B.R. et al. Mitigating thermal mechanical damage potential during twophoton dermal imaging. J. Biomed. Opt. 9, 1265-1270 (2004).

13. Gustafsson, M.G.L. Nonlinear structured-illumination microscopy: Wide-field fluorescence imaging with theoretically unlimited resolution. Proc. Natl. Acad. Sci. USA 102, 13081-13086 (2005).

14. Eggeling, C., Widengren, J., Rigler, R. \& Seidel, C.A.M. Photostability of fluorescent dyes for single-molecule spectroscopy: mechanisms and experimental methods for estimating photobleaching in aqueous solution. in Applied fluorescence in chemistry, biology and medicine. (eds., Rettig, W., Strehmel, B., Schrader, M. \& Seifert, H.) 193-240 (Springer, Berlin, 1999).

15. Tsien, R.Y. The green fluorescent protein. Annu. Rev. Biochem. 67, 509-544 (1998)

16. Sanchez, E.J., Novotny, L., Holtom, G.R. \& Xie, X.S. Room-temperature fluorescence imaging and spectroscopy of single molecules by two-photon excitation. J. Phys. Chem. A 101, 7019-7023 (1997).

17. Eggeling, C., Widengren, J., Rigler, R. \& Seidel, C.A.M. Photobleaching of fluorescent dyes under conditions used for single-molecule detection: evidence of two-step photolysis. Anal. Chem. 70, 2651-2659 (1998).

18. Widengren, J., Mets, Ü. \& Rigler, R. Fluorescence correlation spectroscopy of triplet states in solution: A theoretical and experimental study. J. Phys. Chem. 99, 13368-13379 (1995).

19. Patterson, G.H. \& Piston, D.W. Photobleaching in two-photon excitation microscopy. Biophys. J. 78, 2159-2162 (2000).

20. Eggeling, C., Volkmer, A. \& Seidel, C.A.M. Molecular photobleaching kinetics of rhodamine $6 \mathrm{G}$ by one- and two-photon induced confocal fluorescence microscopy. ChemPhysChem 6, 791-804 (2005).

21. Kasha, M. Paths of molecular excitation. Radiat. Res. 2 (Suppl.), 243-275 (1960).

22. Koester, H.J., Baur, D., Uhl, R. \& Hell, S.W. $\mathrm{Ca}^{2+}$ fluorescence imaging with pico- and femtosecond two-photon excitation: signal and photodamage. Biophys. J. 77, 2226-2236 (1999).

23. Nagai, T. et al. A variant of yellow fluorescent protein with fast and efficient maturation for cell-biological applications. Nat. Biotechnol. 20, 87-90 (2002). 